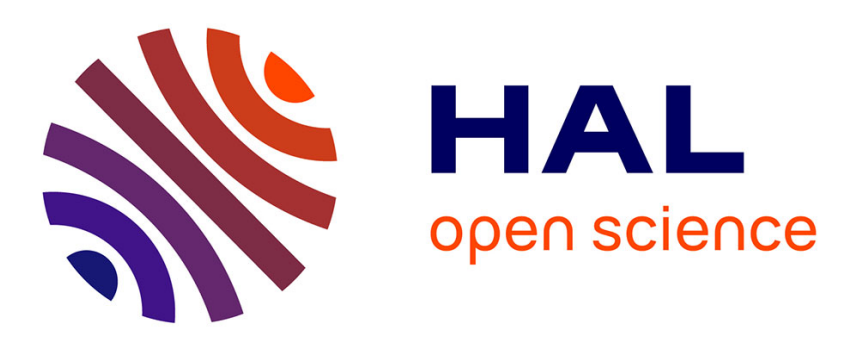

\title{
Drag-model sensitivity of Kelvin-Helmholtz waves in canopy flows
}

Nicola Luminari, Christophe Airiau, Alessandro Bottaro

\section{To cite this version:}

Nicola Luminari, Christophe Airiau, Alessandro Bottaro. Drag-model sensitivity of Kelvin-Helmholtz waves in canopy flows. Physics of Fluids, 2016, vol. 28 ( $\left.\mathrm{n}^{\circ} 12\right)$, p. $124103.10 .1063 / 1.4971789$. hal-01597197

\author{
HAL Id: hal-01597197 \\ https://hal.science/hal-01597197
}

Submitted on 28 Sep 2017

HAL is a multi-disciplinary open access archive for the deposit and dissemination of scientific research documents, whether they are published or not. The documents may come from teaching and research institutions in France or abroad, or from public or private research centers.
L'archive ouverte pluridisciplinaire HAL, est destinée au dépôt et à la diffusion de documents scientifiques de niveau recherche, publiés ou non, émanant des établissements d'enseignement et de recherche français ou étrangers, des laboratoires publics ou privés. 


\section{OATAO \\ Open Archive Toulouse Archive Ouverte}

\section{Open Archive Toulouse Archive Ouverte (OATAO)}

OATAO is an open access repository that collects the work of Toulouse researchers and makes it freely available over the web where possible.

This is an author-deposited version published in: http://oatao.univ-toulouse.fr/ Eprints ID: 18315

To link to this article : DOI: $10.1063 / 1.4971789$

URL : http://dx.doi.org/10.1063/1.4971789

To cite this version: Luminari, Nicola and Airiau, Christophe and Bottaro, Alessandro Drag-model sensitivity of Kelvin-Helmholtz waves in canopy flows. (2016) Physics of Fluids, vol. 28 ( $\left.\mathrm{n}^{\circ} 12\right)$. p. 124103. ISSN 1070-6631

Any correspondence concerning this service should be sent to the repository administrator:staff-oatao@listes-diff.inp-toulouse.fr 


\section{Drag-model sensitivity of Kelvin-Helmholtz waves in canopy flows}

Nicola Luminari, Christophe Airiau, and Alessandro Bottaro

Citation: Phys. Fluids 28, 124103 (2016); doi: 10.1063/1.4971789

View online: http://dx.doi.org/10.1063/1.4971789

View Table of Contents: http://aip.scitation.org/toc/phf/28/12

Published by the American Institute of Physics

\section{Articles you may be interested in}

Aerodynamics of two-dimensional flapping wings in tandem configuration

Phys. Fluids 28, 121901121901 (2016); 10.1063/1.4971859

The minimal flow unit in complex turbulent flows

Phys. Fluids 28, 125102125102 (2016); 10.1063/1.4968827

Finding order in complexity: A study of the fluid dynamics in a three-dimensional branching network

Phys. Fluids 28, 123602123602 (2016); 10.1063/1.4971315

On the flow structures and hysteresis of laminar swirling jets

Phys. Fluids 28, 123604123604 (2016); 10.1063/1.4972227

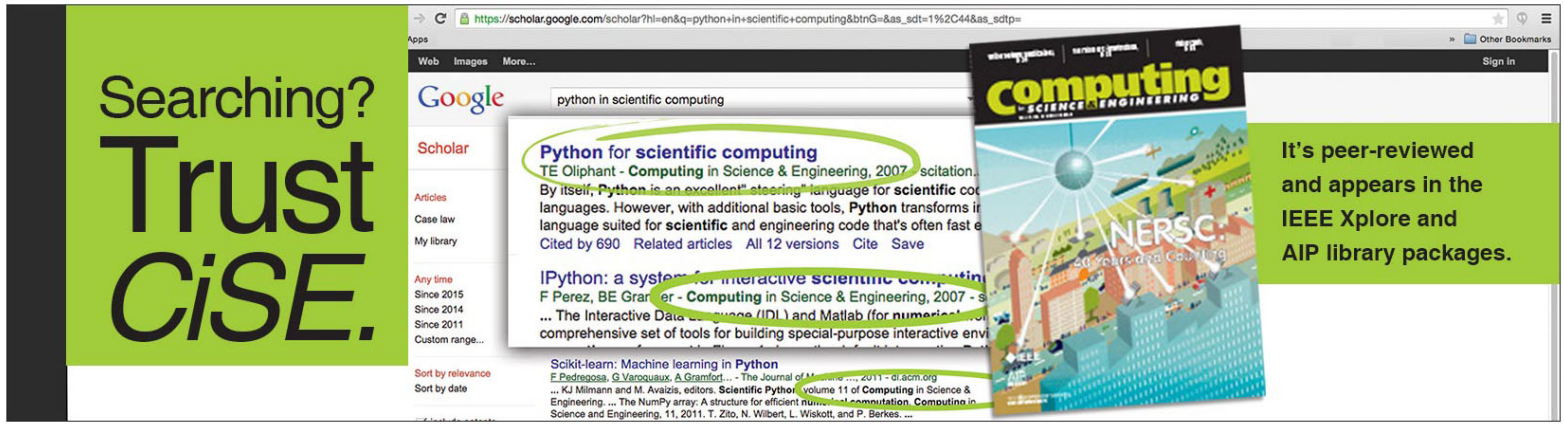




\title{
Drag-model sensitivity of Kelvin-Helmholtz waves in canopy flows
}

\author{
Nicola Luminari, Christophe Airiau, and Alessandro Bottaro ${ }^{a)}$ \\ Université de Toulouse, Institut de Mécanique des Fluides de Toulouse, UMR 5502, \\ Allée du Pr. Camille Soula, F31400 Toulouse, France
}

(Received 28 July 2016; accepted 22 November 2016; published online 16 December 2016)

\begin{abstract}
Two models of the flow over and through an immersed, vegetated layer are examined to study the onset of instability waves across the layer and to assess the effect of mild variations in the mean flow and in the drag force exerted by the canopy onto the frequency and growth rate of the monami instability. One of the two models, based on the use of Darcy's equation, with a tensorial permeability, within the canopy is more robust than the other (which uses a scalar drag coefficient), i.e., it is less sensitive to the inevitable imperfections or approximations in the input data. Published by AIP Publishing. [http://dx.doi.org/10.1063/1.4971789]
\end{abstract}

\section{INTRODUCTION}

Flows through submerged aquatic plants exhibit large scale vortices at the top of the vegetation, advecting along the flow direction and causing a periodic waving of the plants, referred to as monami. ${ }^{1}$ Vortices arise from the nonlinear amplification of a Kelvin-Helmholtz instability mode, related to the presence of an inflection point in the base flow profile $;^{2}$ the profile itself is inflectional because the fluid is slowed down by the drag exerted by the canopy, whose modeling has recently been addressed..$^{3-5}$ The correct prediction of the onset and characteristics of the Kelvin-Helmholtz instability is important for assessing the effects of turbulence, in particular to

- understand how the vertical exchange of momentum occurs, ${ }^{6}$

- clarify how the transport of $\mathrm{CO}_{2}$, dissolved nutrients or sediments takes place between the obstructed vegetation flow and the free overflow motion, ${ }^{7-10}$ and also

- assess the changes in the morphology of the vegetation in inland or coastal wetlands in response to continuous periodic forcing. ${ }^{11,12}$

Because of the flexibility of the vegetation, some theoretical studies have focussed on the modeling of the stems of the aquatic plants and their displacement in response to the forcing by the water flow. ${ }^{3,13}$ However, Kelvin-Helmholtz vortices occur whether or not the plants bend and-to ascertain causes and effects to first order - it is acceptable to focus on the flow over and through a submerged array of rigid, cylindrical pillars. This has been the basis of the approach by Ghisalberti and $\mathrm{Nepf}^{14-16}$ who have conducted a series of careful experiments; their results have often been used by fluid dynamicists to put forth and test theoretical hypotheses to predict the frequency and wavelength of the large scale vortical motion, for a variety of conditions. The configuration studied consists of a regular grid of rigid pillars, orthogonal to the surface, of identical height $h$; in some of the theoretical models proposed to analyze the stability of this system, the Rayleigh equation is used throughout the water channel, with or without a drag term in correspondence of the canopy. ${ }^{2-4}$ Zampogna et al. ${ }^{5}$ have recently demonstrated that the addition of a drag term through the vegetation reduces the amplification factor of the Kelvin-Helmholtz instability throughout the whole range of wavenumbers and increases mildly the wavelength of the fastest growing mode; further unpublished work by the same authors shows that the addition of a mixing length turbulence model in the stability equations has but a negligible influence on the leading instability mode. Questions

a)Permanent address: DICCA, Scuola Politecnica, Università di Genova, 1 via Montallegro, 16145 Genova, Italy. 
remain, however, on the accuracy of the drag model and on its sensitivity. A partial answer to these questions is provided in Ref. 5: there, a different model, applicable within the vegetated layer and based on the equations ruling the behavior of a transversely isotropic porous medium, has been developed and the stability results appear to better match experimental correlations. This conclusion is, however, not consolidated yet, and further studies are needed to assess the influence of the model of the drag force through the vegetation, both in setting up a particular (inflectional) mean flow and on the onset and growth of Kelvin-Helmholtz waves.

The present work addresses the points above through an adjoint-based sensitivity analysis along the lines of Bottaro et al. $;^{17}$ the direct stability equations are written with account of viscosity, and the adjoint equations are found and solved in the temporal framework. Results in the spatial setting are discussed in Appendix B, where a digression is made on the computation of the group velocity of the instability waves by the use of the adjoint fields. The sensitivity functions to both mild modifications in the base shear layer and in the drag coefficient are computed and discussed. Finally, a different sensitivity analysis is developed on the basis of the recent anisotropic model by Zampogna et al..$^{5}$ and the results qualitatively compared to those obtained with the more conventional isotropic-drag-force model.

\section{MODEL OF THE CANOPY FLOW}

\section{A. The mean flow}

To obtain the mean flow on top of which small amplitude perturbations are superimposed, the procedure outlined by Ghisalberti and $\mathrm{Nepf}^{15}$ and recently closely followed by Zampogna et al. ${ }^{5}$ is used. For the sake of conciseness, the procedure-which relies on several empirical correlations-is not repeated here, aside from a few brief comments. A mildly inclined water channel is considered, with a canopy formed by rigid cylindrical dowels of height $h$ equal to $13.8 \mathrm{~cm}$ and diameter $d=0.64 \mathrm{~cm}$. The frontal area of the vegetation per unit volume, i.e., the packing density of the elements, is either $a=0.04 \mathrm{~cm}^{-1}$ or $0.08 \mathrm{~cm}^{-1}$; the free surface is positioned at a level $\mathrm{H}=46.7 \mathrm{~cm}$ from the bottom plate and the flow velocity at the free surface, $U_{2}$, varies from 4.4 to $13.7 \mathrm{~cm} / \mathrm{s}$. The Froude number, $F r=\frac{U_{2}}{\sqrt{g H}}$, is thus very low and water surface fluctuations can be ignored. ${ }^{18}$

To a good approximation the mean flow can be taken as steady and parallel, with the streamwise velocity varying from the value $U_{1}$ at the bottom wall (not accounting for the thin bottom boundary layer) to the value $U_{2}$ at the top, near the free surface (cf. Fig. 1). The slope of the bottom surface is very small; it is denoted as $S$ and, in the experiments by Ghisalberti and Nepf, ${ }^{15}$ varies from $1.8 \times 10^{-6}$

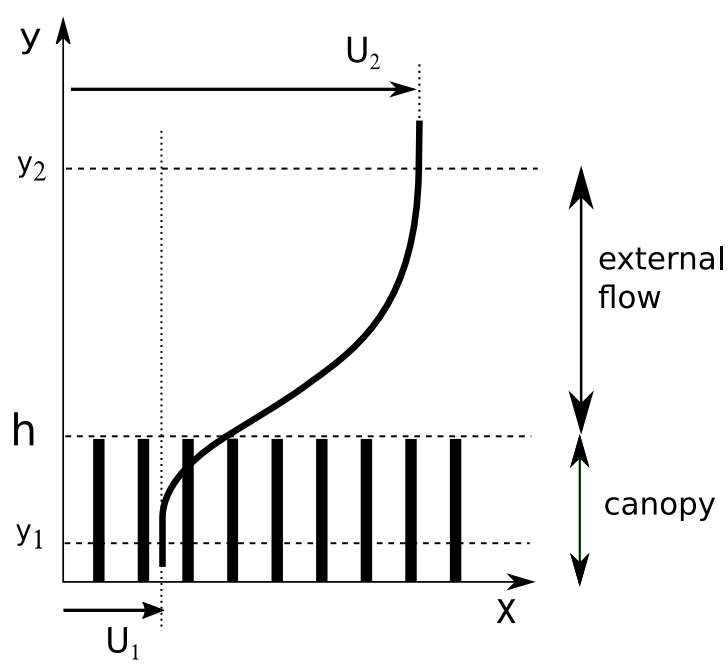

FIG. 1. Configuration studied with main notations. 

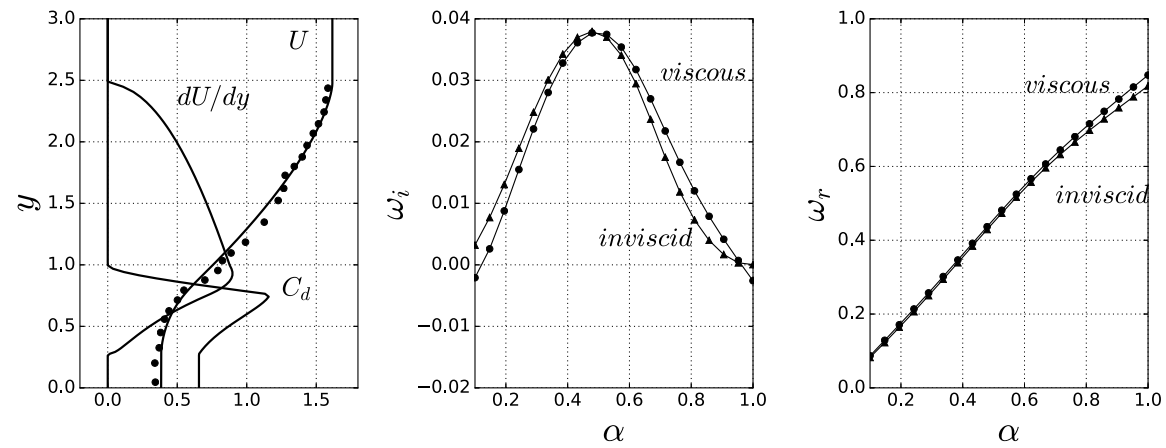

FIG. 2. Left frame: mean flow $U$, together with experimental data points, ${ }^{15}$ its first derivative, and drag coefficient distribution (case G). Center: viscous and inviscid growth rates, $\omega_{i}$, as a function of the streamwise wavenumber $\alpha$. Right: corresponding frequencies, $\omega_{r}$.

to $10^{-4}$; such a slope provides the driving force for the motion. The viscous term is small compared to the turbulent diffusion term, so that the mean streamwise momentum equation can be approximated by

$$
g S=\frac{\partial \overline{u^{\prime} v^{\prime}}}{\partial y}+\frac{1}{2} C_{d} a U^{2}
$$

with $g$ the acceleration of gravity and $C_{d}$ an isotropic drag function available from the experiments, variable across the canopy and equal to zero when $y \geq h$. The Reynolds stress $-\overline{u^{\prime} v^{\prime}}$ is modelled with the Boussinesq assumption, introducing a turbulent viscosity which depends on a mixing length and on the gradient of the mean velocity $U$. Referring to Ghisalberti and $\mathrm{Nepf}^{15}$ for details of the empirical correlations used to close the equations and the solution method, we limit ourselves here to stating that the results obtained for the mean flow are very close to those reported in Zampogna et al. ${ }^{5}$ (cf. their Figure 3) and closely match experimental points for the cases G, H, I, and J considered (we use the same terminology of Ghisalberti and $\mathrm{Nepf}^{14-16}$ to indicate the different flow configurations). An example of mean flow is reported in Fig. 2 (left frame). There, one can observe the computed flow (against discrete measurement points), its first derivative, and the drag coefficient distribution for one representative case (experiment $\mathrm{G}$ ), used below also to discuss stability and sensitivity results.

Other procedures have been employed in the past to calculate the mean flow, with satisfactory results. For example, Singh et al. ${ }^{4}$ have considered a constant value of $C_{d}$ through the canopy, while Zampogna and Bottaro ${ }^{5}$ have coupled, at a fictitious interface, the fluid equations outside the canopy to Darcy's law within the vegetation. Thus, for the purposes of the present paper, the mean flow is assumed as given; it could be, for example, simply a fit through experimental data. Nonetheless, in Appendix A we provide some considerations on how $C_{d}$ affects the mean flow in the model used here.

\section{B. Stability and sensitivity equations} form

A temporal linear stability analysis is carried out, with the generic perturbation $q^{\prime}(x, y, t)$ of the

$$
q^{\prime}(x, y, y, t)=q(y) \mathrm{e}^{i(\alpha x-\omega t)},
$$

with $\alpha$ the real streamwise wavenumber and $\omega$ a complex number whose real part, $\omega_{r}$, is the frequency of the mode and the imaginary part, $\omega_{i}$, is the growth rate. The dimensionless linear stability equations in primitive variables read

$$
\begin{gathered}
i \alpha u+D v=0, \quad D=\mathrm{d} / \mathrm{d} y, \\
{\left[i(\alpha U-\omega)-\frac{D^{2}-\alpha^{2}}{\operatorname{Re}}+a C_{d} U\right] u+U^{\prime} v+i \alpha p=0, \quad U^{\prime}=\frac{\mathrm{d} U}{\mathrm{~d} y},} \\
{\left[i(\alpha U-\omega)-\frac{D^{2}-\alpha^{2}}{R e}\right] v+D p=0,}
\end{gathered}
$$


with the perturbation velocity components which vanish when $y=0$ and $y_{\infty}$. The upper boundary of the computational domain is taken far enough away from the lower boundary to ensure that the results do not vary upon modifications of $y_{\infty}$. All the terms in the equations are dimensionless; the mean speed through the shear layer, $U_{m}=\frac{U_{2}+U_{1}}{2}$, is used to scale the disturbance velocity components, pressure is scaled with $\rho U_{m}^{2}$, distances with $h$, and time with $h / U_{m}$. The Reynolds number in the equations above is thus defined as $R e=\frac{\rho U_{m} h}{\mu}$, with $\rho$ and $\mu$ the fluid's density and dynamic viscosity, respectively. The computations are performed both at the $R e$ values of the experiments and in the inviscid limit $\left(R e^{-1} \rightarrow 0\right)$, for comparison purposes. In the latter case, the boundary conditions are simply $v=0$ at $y=0$ and $y_{\infty}$.

System (1) above and its boundary conditions are, in the following, also written in short notation as $\mathscr{L} q=0$. The eigenvalues of the system are those complex values of $\omega$ which yield non-trivial solutions for $u, v$, and $p$. Two numerical collocation codes are written, and successfully compared; one is based on the equations in primitive variables form, the second solves an Orr-Sommerfeld-like equation (with the addition of the drag term) along the lines of Singh et al. ${ }^{4}$ In both cases, a spectral scheme based on $N$ Chebyshev polynomials is used ( $N$ is typically equal to 300 to ensure grid-converged results), with an algebraic mapping between the physical and the spectral domains (cf. Canuto et al. ${ }^{19}$ ).

Viscous and inviscid stability results for case G are shown in Fig. 2 (center and right frames); differences are small, in consideration of the fact that the Reynolds number of the viscous case is relatively large $(R e=3450)$. The viscous wavenumber of largest amplification is found for $\alpha=0.4790$; the waves are weakly dispersive, particularly at low wavenumbers (an original interpretation of phase and group velocities is proposed in Appendix B). The wavelength of largest growth is smaller than that found by Zampogna et al. ${ }^{5}$ which was 0.73 ; this is related to the slightly different base flow in the two cases (in the present contribution a smoothing has been applied to the $U$ velocity distribution to render $\mathrm{d} U / \mathrm{d} y$ continuous across $y$ ) and highlights the sensitivity of this stability problem to base flow variations.

Following Bottaro et al., ${ }^{17}$ it is assumed that small variations in base flow and drag coefficient entail infinitesimal variations in the system's eigenvalues and eigenfunctions. We stress here the fact that $C_{d}$ is identically equal to zero outside of the canopy, and this implies that there are no possible variations in $C_{d}$ for $y \geq 1$. The sensitivity functions to variations in $U$ and $C_{d}$ are obtained by using the properties of the adjoint system which is defined from the Lagrange identity

$$
\left\langle q^{\dagger}, \mathscr{L} q\right\rangle=\left\langle\mathscr{L}^{\dagger} q^{\dagger}, q\right\rangle+[B T]_{0}^{y_{\infty}} .
$$

The boundary terms $B T$ are set to zero by the appropriate choice of the boundary conditions for the adjoint problem, and the inner product is defined as

$$
\langle f, g\rangle=\int_{0}^{y_{\infty}} \bar{f}(y) g(y) d y,
$$

with the overbar denoting complex conjugation. The adjoint system of equations, $\mathscr{L}^{\dagger} q^{\dagger}=0$, in full form reads

$$
\begin{gathered}
i \alpha u^{\dagger}+D v^{\dagger}=0, \\
{\left[i\left(\alpha U-\omega^{\dagger}\right)+\frac{D^{2}-\alpha^{2}}{R e}-a C_{d} U\right] u^{\dagger}+i \alpha p^{\dagger}=0,} \\
{\left[i\left(\alpha U-\omega^{\dagger}\right)+\frac{D^{2}-\alpha^{2}}{R e}\right] v^{\dagger}+D p^{\dagger}-U^{\prime} u^{\dagger}=0,}
\end{gathered}
$$

with the same boundary conditions as in the direct system. The adjoint eigenvalue $\omega^{\dagger}$ is the complex conjugate of the direct eigenvalue $\omega ; u^{\dagger}, v^{\dagger}$, and $p^{\dagger}$ are the eigenmodes of the adjoint problem.

The sensitivity functions are obtained by imposing a small variation in the inner product as

$$
0=\delta\left\langle q^{\dagger}, \mathscr{L} q\right\rangle=\left\langle q^{\dagger}, \mathscr{L} \delta q\right\rangle+\left\langle q^{\dagger}, \frac{\partial \mathscr{L}}{\partial U} q \delta U\right\rangle+\left\langle q^{\dagger}, \frac{\partial \mathscr{L}}{\partial C_{d}} q \delta C_{d}\right\rangle+\left\langle q^{\dagger}, \frac{\partial \mathscr{L}}{\partial \omega} q\right\rangle \delta \omega,
$$



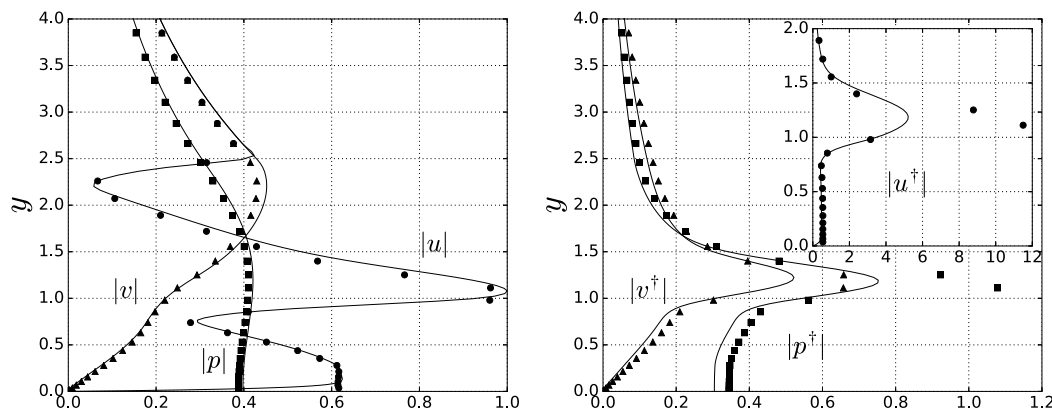

FIG. 3. Moduli of direct (left frame) and adjoint (right frame) eigenfunctions for the viscous (continuous lines, $R e=3450$ ) and the inviscid (symbols) case, in correspondence to the wavenumber of largest amplification.

and considering the effect of independent variations of $U$ and $C_{d}$ onto $q$ and $\omega$. It is found that

$$
\delta \omega=\delta \omega_{r}+i \delta \omega_{i}=\int_{0}^{y_{\infty}} G_{U}(y) \delta U(y) d y+\int_{0}^{1} G_{C_{d}}(y) \delta C_{d}(y) d y,
$$

with

$$
\begin{aligned}
G_{U} & =\alpha\left[\overline{v^{\dagger}} v+\overline{u^{\dagger}} u\right]+i\left(\overline{u^{\dagger}} v\right)^{\prime}-i a C_{d} \overline{u^{\dagger}} u \quad \text { and } \\
G_{C_{d}} & =-i a U \overline{u^{\dagger}} u
\end{aligned}
$$

the required sensitivity functions; the real parts of $G_{U}$ and $G_{C_{d}}$ express sensitivities to variations in the frequency of the mode while the imaginary parts are sensitivities to variations in the growth rate. Direct and adjoint eigenfunctions are normalized so that $N_{\omega}=1$, with

$$
N_{\omega}=\int_{0}^{y_{\infty}}\left[\overline{v^{\dagger}} v+\overline{u^{\dagger}} u\right] d y .
$$

An example of direct and adjoint eigenfunctions is provided in Fig. 3, both in the viscous case $(R e=3450)$ and in the inviscid limit, for $\alpha=0.4790$. It is interesting to observe that while the direct eigenfunctions are almost overlapped, the same is not the case for the adjoint eigenfunctions, with the inviscid mode (drawn with symbols) which has a larger amplitude than the viscous one. The shapes of the direct eigenfunctions are very close to those reported in Ref. 5. The adjoint modes reveal that the flow is most sensitive to streamwise forcing, particularly when it occurs slightly above the edge of the canopy. Source terms in the mass conservation and in the vertical momentum equations are much less effective.

\section{SENSITIVITY RESULTS FOR THE ISOTROPIC DRAG MODEL}

Some representative sensitivity functions are plotted in Fig. 4; viscous and inviscid results concur in showing that the largest sensitivities to variations of $U$ are found right above the vegetation's edge, where there are peaks in the adjoint eigenfunctions and where $\mathrm{d}^{2} U / \mathrm{d} y^{2}$ vanishes. The $U$-sensitivities are negligible within the vegetated layer and for values of $y$ larger than twice the canopy's height. The $C_{d}$-sensitivities are non-negligible only in close proximity of the interface.

It is interesting to observe that real and imaginary parts of the $U$-sensitivity functions are shifted in $y$ with respect to one another; this means that, for example, a localized perturbation at a given $y$ position (above the canopy) might have a strong repercussion on the growth rate but not on the frequency of the most unstable Kelvin-Helmholtz mode, or vice versa. Comparing left and right frames of the figure, it is seen that inviscid $G_{U}$ sensitivity functions display sharper peaks and steeper gradients, and yield larger variations in $\omega$ than their viscous counterparts in the proximity of the $U$ inflection point, a clear consequence of the inviscid mechanism ruling the instability. In both the viscous and the inviscid models, the sensitivity to base flow variations is typically one order of magnitude larger than the sensitivity to changes in the drag coefficient. 

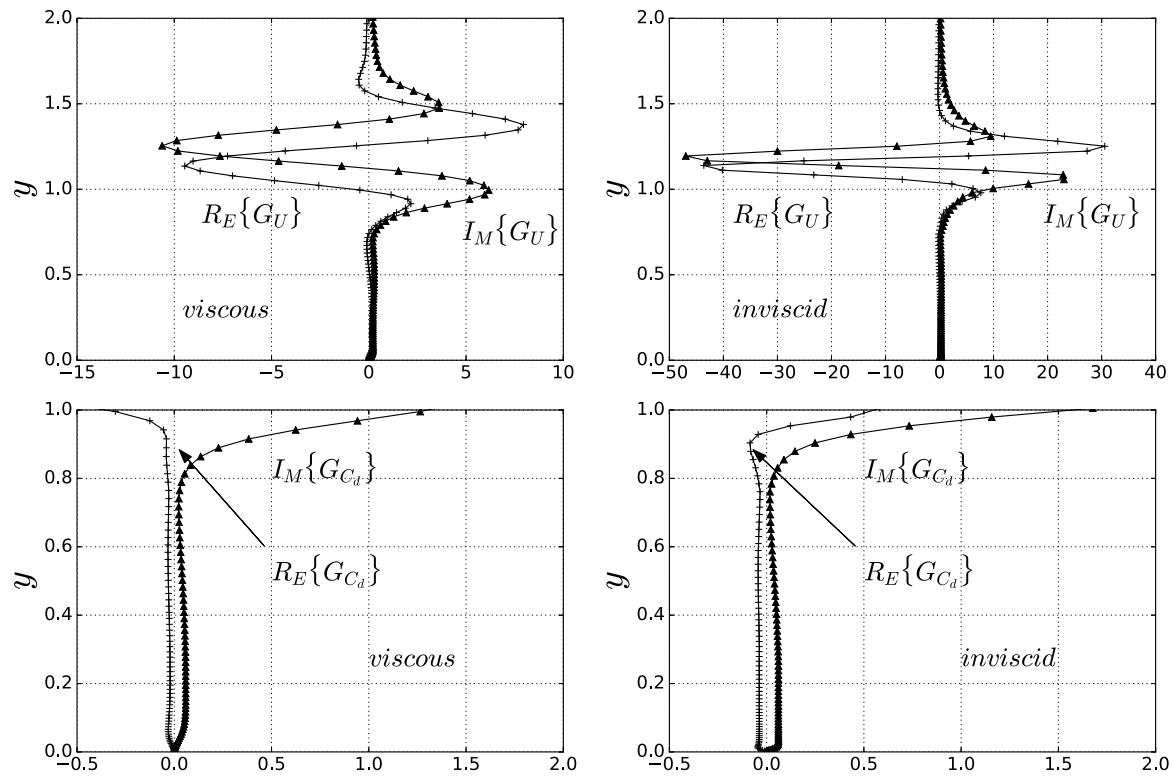

FIG. 4. Real and imaginary parts of the sensitivities to mean flow variations (top) and to variations in the drag distribution function (bottom), for the parameters of Fig. 3.

The infinite norm of the sensitivities for the four cases studied $(\mathrm{G}, \mathrm{H}, \mathrm{I}$, and $\mathrm{J})$ is reported in Fig. 5; the main result found is that $\left|G_{U}\right|_{\infty}$ grows monotonically with $\alpha$ (and more so in the inviscid case) whereas $\left|G_{C_{d}}\right|_{\infty}$ does not. It is consistently found that $\left|G_{U}\right|_{\infty}$ of case $\mathrm{H}$ is larger than that of case I, which exceeds the corresponding value of case $\mathrm{J}$, in turn larger than $\left|G_{U}\right|_{\infty}$ of case $\mathrm{G}$. This is not unexpected in view of the values of the mean shear $\frac{U_{2}-U_{1}}{H}$ which are, going from $\mathrm{H}$ to $\mathrm{G}$, equal
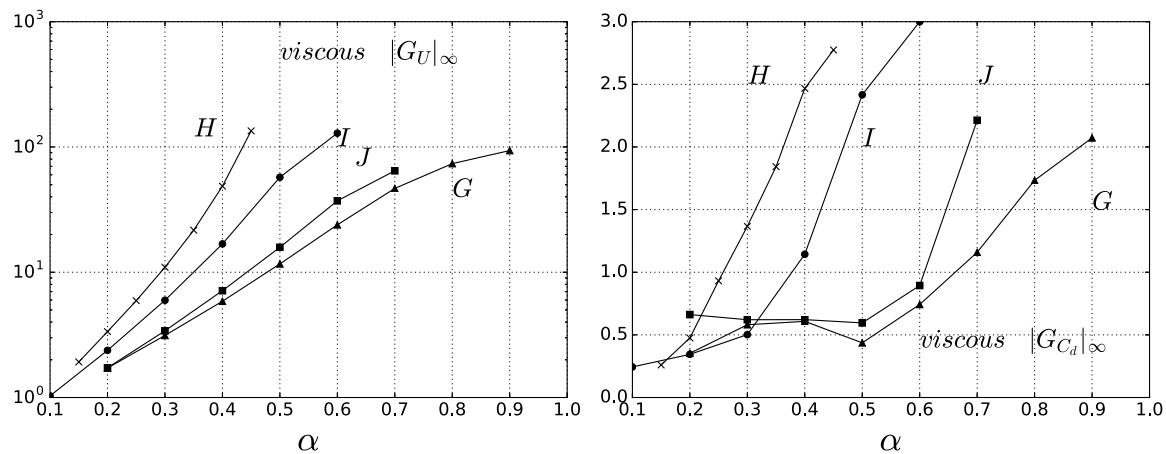

$\alpha$
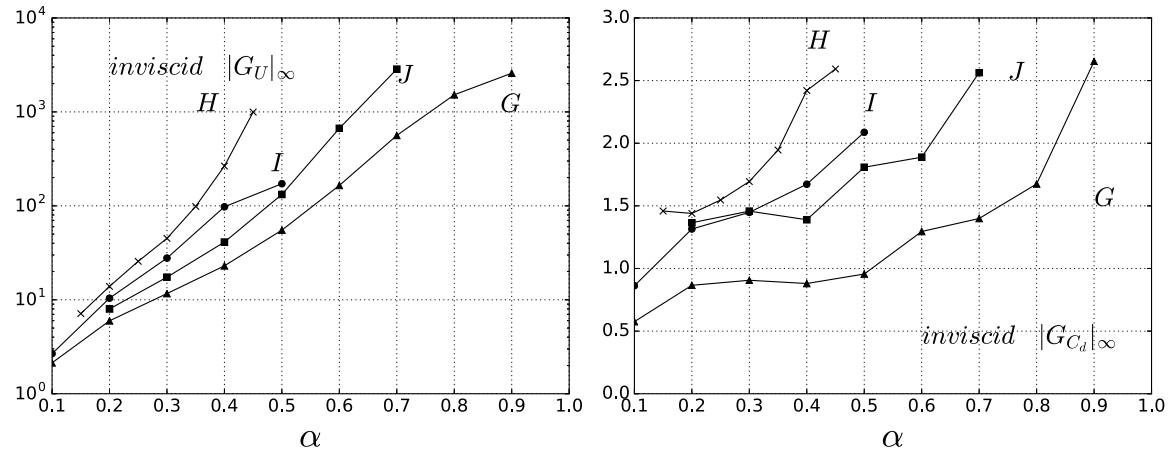

FIG. 5. Infinite norms of the sensitivity functions for varying $\alpha$. 
to $0.236,0.158,0.084$, and $0.071 \mathrm{~s}^{-1}$, respectively. The sensitivity of the eigenvalue $\omega$ to variations in the mean flow is generally stronger than the corresponding sensitivity to variations in the drag coefficient (aside for the long wave limit, where they are comparable). This might be interpreted positively, considering that the use of a scalar coefficient $C_{d}$ to represent the drag within the canopy is but a crude approximation. An alternative model to represent the flow throughout a network of rigid, cylindrical dowels has recently been proposed by Zampogna et al. ${ }^{5}$ The sensitivity results for such a new model are discussed next.

\section{AN ALTERNATIVE SENSITIVITY MODEL: ACCOUNTING FOR THE CANOPY ANISOTROPICITY}

The stability problem in this section is based on the coupling between two regions, one outer region dominated by inertia and ruled by the inviscid equations and an inner one dominated by viscosity and ruled by Darcy's law, with account of the canopy geometry through a tensorial permeability, as described by Zampogna et al..$^{5}$ Normalizing the disturbance equation which couples pressure and velocity in the inner region with the same scales as previously, we obtain

$$
u_{i}^{\prime}=-\operatorname{Re} \frac{d}{a h^{2}} \mathcal{K}_{i j} \frac{\partial p^{\prime}}{\partial x_{j}}, \quad\left(x_{1}, x_{2}\right)=(x, y),
$$

with $\mathcal{K}_{i j}$ the dimensionless permeability. The effective interface between the inertial region and the slow, viscosity-dominated region does not coincide with the edge of the canopy; in fact, the rapid outer flow penetrates through the upper part of the vegetation and an effective matching between outer and inner flows must be enforced some distance $\delta$ below the canopy's edge. ${ }^{20}$ This distance, a penetration depth, has been successfully computed by Zampogna and Bottaro ${ }^{21}$ for a few cases and is found to increase with the Reynolds number of the flow; for experiment $\mathrm{G}$ discussed below it is $\delta \approx 0.40 .{ }^{22}$ On account of the results shown in Fig. 4, with the sensitivities which are negligible for $y \approx 0.60$, we expect that the exact position of the effective interface will not affect the results significantly.

Using the fact that the velocity within the orthotropic porous medium is divergence free, the interface condition to be applied at $y_{\text {itf }}=1-\delta$ is found to be

$$
\left.v\right|_{i f f}+\left.B(\alpha) p\right|_{i f f}=0
$$

with

$$
B=\operatorname{Re} \frac{d}{a h^{2}} \sqrt{\mathcal{K}_{11} \mathcal{K}_{22}} \alpha \tanh \theta \quad \text { and } \quad \theta=\alpha \sqrt{\frac{\mathcal{K}_{11}}{\mathcal{K}_{22}}} y_{i t f} .
$$

The second boundary condition that the Rayleigh stability equation must satisfy at $y_{\infty}$ is simply $v=0$. Thus, we solve only for the inviscid flow in the outer region, and the permeability of the inner domain enters the equations only through the interface condition (5). $\mathcal{K}_{i j}$ is a twoby-two diagonal tensor; $\mathcal{K}_{11}$ is the component of the dimensionless permeability along $x$ and $\mathcal{K}_{22}$ is the $y$ component. For case $\mathrm{G}$ considered here, the packing density of the elements is $a=0.04 \mathrm{~cm}^{-1}$; it is also found that $\mathcal{K}_{11}=0.0512$ and $\mathcal{K}_{22}=0.0575,{ }^{22}$ so that the function $B(\alpha)$ reads $B=15.727 \alpha \tanh (0.566 \alpha)$.

\section{A. The sensitivity equations}

The adjoint equations in this case are the same as system (2), without the terms containing $1 / R e$ and $C_{d}$, and the boundary conditions are

$$
\left.v^{\dagger}\right|_{i t f}-\left.B(\alpha) p^{\dagger}\right|_{i f f}=0,\left.\quad v^{\dagger}\right|_{y_{\infty}}=0 .
$$

The variation in the complex frequency is related to variations in the mean flow and in the permeability components through the equation

$$
\delta \omega=\int_{y_{i f f}}^{y_{\infty}} G_{U}(y) \delta U(y) d y+G_{\mathcal{K}_{11}} \delta \mathcal{K}_{11}+G_{\mathcal{K}_{22}} \delta \mathcal{K}_{22},
$$



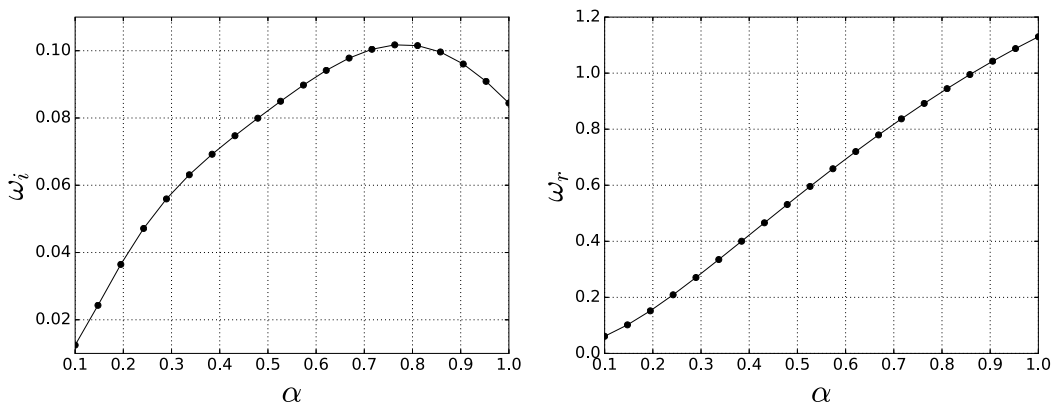

FIG. 6. Amplification factor (left) and frequency of the most unstable mode as a function of $\alpha$, for the anisotropic drag model.

with

$$
\begin{gathered}
G_{U}=\alpha\left[\overline{v^{\dagger}} v+\overline{u^{\dagger}} u\right]+i\left(\overline{u^{\dagger}} v\right)^{\prime}, \\
G_{\mathcal{K}_{11}}=-\frac{i}{2} \alpha \operatorname{Re} \frac{d}{a h^{2}}\left[p \overline{p^{\dagger}}\right]_{i t f} \sqrt{\frac{\mathcal{K}_{22}}{\mathcal{K}_{11}}}\left\{\tanh \theta+\frac{\theta}{\cosh ^{2} \theta}\right\}, \quad \text { and } \\
G_{\mathcal{K}_{22}}=-\frac{i}{2} \alpha \operatorname{Re} \frac{d}{a h^{2}}\left[p \overline{p^{\dagger}}\right]_{i t f} \sqrt{\frac{\mathcal{K}_{11}}{\mathcal{K}_{22}}}\left\{\tanh \theta-\frac{\theta}{\cosh ^{2} \theta}\right\}
\end{gathered}
$$

the required sensitivities, with the normalization $\int_{y_{i f}}^{y_{\infty}}\left[\overline{v^{\dagger}} v+\overline{u^{\dagger}} u\right] d y=1$. In writing $\delta \omega$ above, we have made the assumption that the mean flow $U$ does not vary at the two extreme points of the integration domain.

The stability results (for the same parameters as in Fig. 2) are displayed in Fig. 6. As already observed in Ref. 5, both the growth rate and the frequency are slightly larger with this model than with the isotropic resistance model, for all $\alpha$ 's, and the most unstable mode is found at a larger value of $\alpha$ (here $\alpha \approx 0.8$ ) in better agreement with experimental correlations. ${ }^{2,5}$ Also in this case the waves are found to be only weakly dispersive.

Eigenfunctions are plotted in Fig. 7, together with the real and imaginary parts of the $G_{U}$ sensitivity function. As in Fig. 3, the modulus of the $u$ eigenfunction peaks near the edge of the canopy $(y=1)$, whereas the adjoint eigenfunctions have a maximum value slightly above. As a general remark, the shapes of the direct and adjoint modes are quite similar to those found with the isotropic resistance model; as reported at the end of Section II B, it is found that the flow is most sensitive to streamwise momentum forcing. Also, real and imaginary parts of $G_{U}$ have a double-peak structure, like in the isotropic-drag model, but now the largest absolute value of $G_{U}$ is
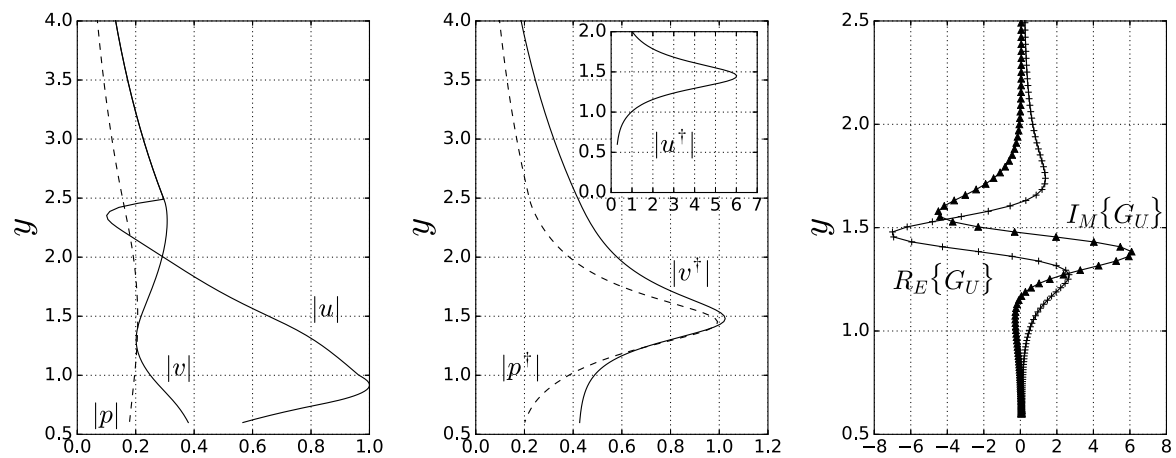

FIG. 7. Left and center frames: moduli of direct and adjoint eigenfunctions; pressure and "adjoint pressure" are drawn with dashed lines. Right: real and imaginary parts of the sensitivity function $G_{U}(\alpha=0.4790)$. 

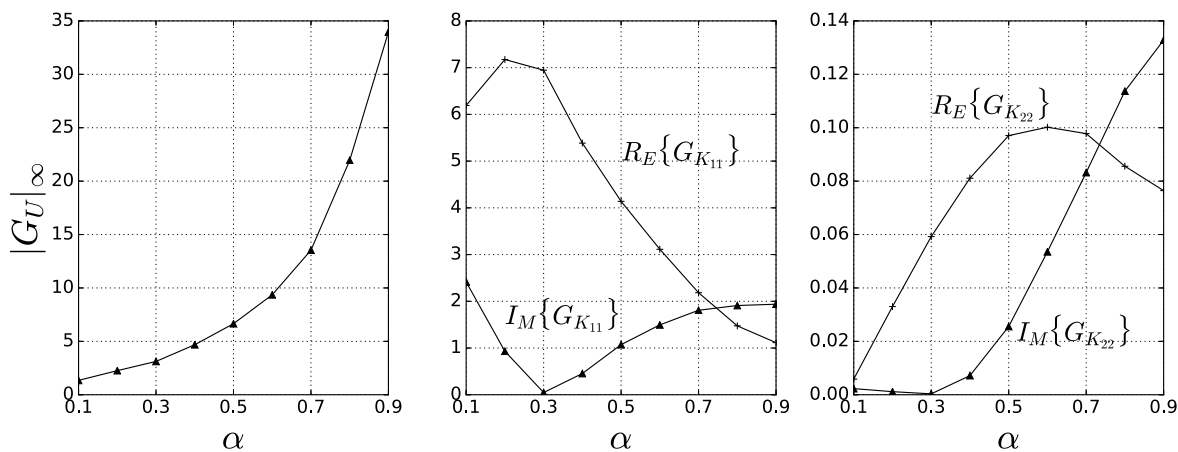

FIG. 8. Case G. Left: infinite norm of $G_{U}$ for varying $\alpha$. Center and right frames: real and imaginary parts of the sensitivity coefficients to variations in the permeability components.

smaller and shifted towards a larger $y$ than in the previous inviscid case (cf. Fig. 4, top-right frame). This can also be appreciated by the inspection of Fig. 8 (left); $\left|G_{U}\right|_{\infty}$ still grows monotonically with $\alpha$, but the sensitivity is smaller than that computed earlier (cf. Fig. 5) with either the viscous or inviscid model (it is actually closer to the viscous sensitivity, as an effect of the interface condition). Furthermore, it is interesting to observe that both real and imaginary parts of $G_{U}$ vanish for $y=\left.y\right|_{\text {itf }}$ (cf. Fig. 7, right), and this supports the statement made previously that a small shift in the position of the effective interface has but a minor influence on the most unstable mode.

The sensitivity coefficients for the two components of the permeability tensors are displayed in Fig. 8 (center and right frames): the present model is more effective to variations in $\mathcal{K}_{11}$ than to $\delta \mathcal{K}_{22}$ as far as modifying the complex eigenfrequency. Significantly, different ranges of wavenumbers behave differently as far as the variation in $\omega$ is concerned. The frequency $\omega_{r}$ of long waves (around $\alpha \approx 0.3$ ) is more easily modified by acting on $\mathcal{K}_{11}$ (with an almost negligible effect on the growth rate of the wave); conversely, the growth rate of modes with large values of $\alpha$ is affected efficiently by variations in the first component of the permeability tensor.

\section{CONCLUDING REMARKS}

We have considered two different models of the flow through a vegetated layer experiencing Kelvin-Helmholtz destabilization. One model is based on the use of a single drag coefficient to express the force exerted by the vegetation on the fluid, the second considers the canopy as an orthotropic porous medium and is based on Darcy's equation with a tensorial permeability. ${ }^{21}$ Both models have advantages and drawbacks. The main advantage of the first model is that the drag coefficient can be taken to vary across the canopy; whether this positive consideration, based on macroscopic experimental measurements, ${ }^{14-16}$ carries over to the stability problem remains to be established. The second model, applicable to dense porous media, considers two independent parameters to express the disturbance flow perpendicular and parallel to the rigid dowels forming the canopy. Such parameters and components of the transversely isotropic permeability tensor $\mathcal{K}_{i j}$ arise from the solution of a local Oseen problem. ${ }^{21}$ The drawback of the second model is the fact that an interface (whether real or effective) appears, and adequate matching conditions must be enforced there. Despite much work since the seminal contribution by Beavers and Joseph, ${ }^{23}$ a consensus on the "best" interface conditions between a pure fluid region and a porous medium has not yet emerged.

The models have been put to test through a classical sensitivity analysis. ${ }^{17}$ Beyond displaying stability results which correspond better to those to be expected from available experimental correlations, ${ }^{2,5}$ the anisotropic model is less sensitive to variations in the base flow (with potentially larger variations in frequency and growth rate of the instability mode for the case of shorter waves). As far as a direct comparison between $G_{C_{d}}$ and $G_{\mathcal{K}_{i i}}$ is concerned, this can hardly be made since the variables represent different objects; in particular, the pressure drop through the canopy depends directly on $C_{d}$ and inversely on the permeability. The present results indicate that the anisotropic 
model depends significantly on the value of the apparent ${ }^{21}$ permeability component $\mathcal{K}_{11}$, whose evaluation must thus be conducted carefully. This model is also of interest for further developments, in particular for the study of instabilities developing over waving canopies. Darcy's law in this latter case would need to be modified, as described in Refs. 24 and 25.

\section{ACKNOWLEDGMENTS}

The authors would like to thank the IDEX Foundation of the University of Toulouse for the financial support granted to the last author under the project "Attractivity Chairs." The computations have been conducted at the CALMIP center, Grant No. P1540. The referees are gratefully acknowledged for their comments leading, in particular, to the correct interpretation of the sensitivity of the drag coefficient and to the material in Appendix A.

\section{APPENDIX A: EFFECT OF $c_{d}$ ON THE MEAN FLOW}

In Section II of the paper it is described how the eigenvalue $\omega$ varies as an effect of independent variations of $U$ and $C_{d}$. However, since $C_{d}$ is not zero within the canopy and it is used to compute the mean flow profile $U$, we should in principle have expressed $\delta U$ as $\delta U=\frac{\mathrm{d} U}{\mathrm{~d} C_{d}} \delta C_{d}$ and considered a single sensitivity function $G_{C_{d}}^{*}=G_{C_{d}}+\frac{\mathrm{d} U}{\mathrm{~d} C_{d}} G_{U}$, instead of the two sensitivities given in Equation (4). This would have certainly been the appropriate line of action if the mean flow equation were issued from exact equations, in which case we should have considered also the adjoint of the base flow equation in our variational problem. However, the mean flow model by Ghisalberti and $\mathrm{Nepf}^{15}$ contains empirical approximations and parameters, and alternative models ${ }^{4,5}$ _including very different ones - have been used successfully in the past to predict the mean field; we have thus made the choice, in both Sections III and IV, of considering the mean flow as given, and to take independent variations of $U$ and $C_{d}$ in the stability analysis to assess the effect of modifications in either variable.

If we were to find how much the base flow depends on the drag coefficient in this particular problem, we would need to determine the function $U\left(C_{d}\right)$ and take its derivative. Since both $U$ and $C_{d}$ are functions of the space coordinate $y$, the implicit dependence can be found, and we have plotted it for one case on the left frame of Figure 9. Clearly, the function $U=f\left(C_{d}\right)$ is not single-valued and therefore the derivative can be calculated only over two separate $U$ (or, equivalently, $y$ ) intervals. We have carried out the derivation numerically over each interval, within the range $0.3 \leq y \leq 1$, and the result is reported on the right frame of Figure 9. The filled triangle and circle symbols indicate the two $y$ intervals within the canopy.

We first observe that both the location where $C_{d}$ is maximum and the shape of the function $U=f\left(C_{d}\right)$ are strongly correlated to the drag law $C_{d}(y)$, modeled by Ghisalberti and Nepf ${ }^{15}$
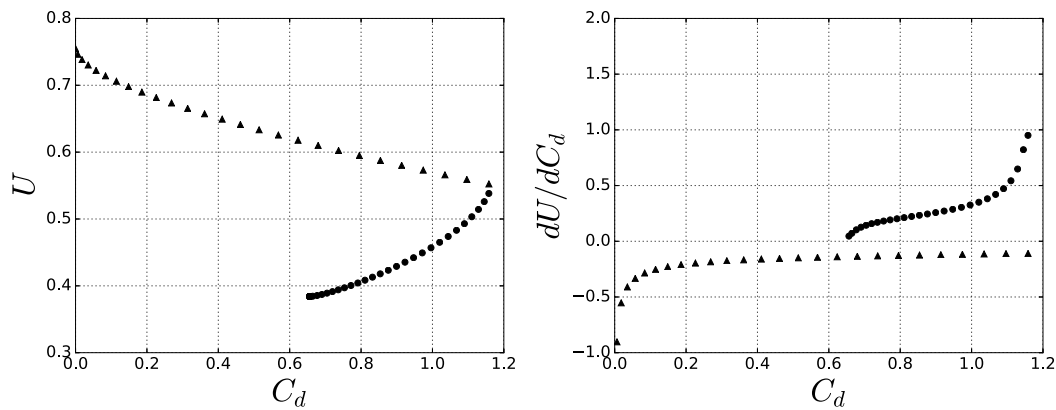

FIG. 9. Case G. Left: mean velocity profile, $U$, versus the drag coefficient, $C_{d}$. Right: first derivative, $\frac{\mathrm{d} U}{\mathrm{~d} C_{d}}$. The triangles denote the region $y \in[0.76,1]$, the filled circles denote the region $y \in[0.3,0.76]$. 
through their measurement data (cf. their Figure 7 and Equation (18)). We also notice that the derivative $\mathrm{d} U / \mathrm{d} C_{d}$ is reasonably small except locally at the point where the derivative of the function is not continuous, where it is of order 1 . The discontinuity there is however artificial since the function $C_{d}(y)$ given in Equation (18) of Ghisalberti and Nepf, ${ }^{15}$ where $C_{d}$ is divided into a parabolic and a linear part, can be easily modified to yield a continuous first derivative at $y=0.76$ if required, still maintaining a mean flow very close to the measured one.

\section{APPENDIX B: A DIGRESSION ON SPATIAL STABILITY THEORY AND GROUP VELOCITY}

Stability problems such as the first one considered in this paper can be approached with the spatial theory framework, with the wavenumber $\alpha$ complex, its imaginary part being a growth rate, and the circular frequency $\omega$ a real constant parameter. Let us generalize the sensitivity analysis by considering, as a first step, $\alpha$ and $\omega$ as complex numbers which can vary. Equation (3) contains one additional term and reads

$$
0=\left\langle q^{\dagger}, \mathscr{L} \delta q\right\rangle+\left\langle q^{\dagger}, \frac{\partial \mathscr{L}}{\partial U} q \delta U\right\rangle+\left\langle q^{\dagger}, \frac{\partial \mathscr{L}}{\partial C_{d}} q \delta C_{d}\right\rangle+\left\langle q^{\dagger}, \frac{\partial \mathscr{L}}{\partial \omega} q\right\rangle \delta \omega+\left\langle q^{\dagger}, \frac{\partial \mathscr{L}}{\partial \alpha} q\right\rangle \delta \alpha .
$$

To obtain the sensitivities in the spatial problem (for which $\delta \omega=0$ ) we now have to solve an adjoint system similar to (2), where $\omega^{\dagger}$ is replaced by $\omega$ and $\alpha$ by $\alpha^{\dagger}$. The variation of the wavenumber $\delta \alpha$ is thus given by

$$
\delta \alpha=\delta \alpha_{r}+i \delta \alpha_{i}=\int_{0}^{y_{\infty}}\left[G_{U}(y) \delta U(y)+G_{C_{d}}(y) \delta C_{d}(y)\right] d y,
$$

the functions $G_{U}$ and $G_{C_{d}}$ maintain the same form as in the temporal theory (4), with the direct and adjoint eigenfunctions which are now normalized by imposing that $N_{\alpha}=-1$, with

$$
N_{\alpha}=\int_{0}^{y_{\infty}}\left[\left(U-\frac{2 i \alpha}{R e}\right)\left(\overline{v^{\dagger}} v+\overline{u^{\dagger}} u\right)+\overline{p^{\dagger}} u+\overline{u^{\dagger}} p\right] d y .
$$

Let us now consider a problem in which $U$ and $C_{d}$ are not allowed to vary, but $\alpha$ and $\omega$ are. With reference to Equation (B1), with any choice of normalization of direct and adjoint modes, it is found that $N_{\omega} \delta \omega=N_{\alpha} \delta \alpha$. Thus, once the adjoint problem is solved, it is possible to accurately compute the group velocity $c_{g}$ of any stability problem using the value of $N_{\omega}$ and $N_{\alpha}$, i.e.,

$$
c_{g}:=\frac{\mathrm{d} \omega_{r}}{\mathrm{~d} \alpha_{r}} \approx \frac{\operatorname{real}\left(N_{\alpha}\right)}{\operatorname{real}\left(N_{\omega}\right)} .
$$

Note that $c_{g}$ above is different from the "complex group velocity" $C_{g}:=\frac{\mathrm{d} \omega}{\mathrm{d} \alpha} \approx \frac{N_{\alpha}}{N_{\omega}}$, and it is also $c_{g} \neq \operatorname{real}\left(C_{g}\right)$. Relation (B2) can be employed in either a spatial or temporal stability analysis and some representative results (for case G) are provided in Table I with the phase velocity $c_{r}:=\omega_{r} / \alpha_{r}$ and the group velocity determined from Equation (B2). The temporal or spatial amplification factors, $\omega_{i}$ or $-\alpha_{i}$, respectively, are also given for all cases using Gaster's transformation: $\omega_{i}=-\alpha_{i} c_{g}$. Two types of errors on the calculation of the group velocity (noted err) are given in the table; the top four values, relative to the temporal theory, are defined as

$$
e r r=\frac{\left|c_{g}\right|_{E q .(\mathrm{B} 2)}-\left.c_{g}\right|_{F D} \mid}{\left.c_{g}\right|_{E q .(\mathrm{B} 2)}},
$$

with $\left.c_{g}\right|_{F D}$ arising from a first-order finite difference approximation of the group velocity. The bottom four values are defined by the formula

$$
\text { err }=\frac{\left|c_{g}\right|_{\text {temporal }}-\left.c_{g}\right|_{\text {spatial }} \mid}{\left.c_{g}\right|_{\text {temporal }}} .
$$

The relative difference on $c_{g}$ between temporal and spatial theory is rather low. It has to be kept in mind, however, that a stability analysis in the spatial framework yields a nonlinear eigenvalue problem, with a consequent larger numerical system than in the temporal framework; therefore, by inverting matrices of the same size, the accuracy is expected to be slightly lower. The accuracy of the growth rate approximated through Gaster's relationship is also found to be acceptable. 
TABLE I. Temporal versus spatial stability, Case G. The model employed here is based on a modified Orr-Sommerfeld equation-rather than a system based on primitive variables as done in the bulk of the paper-which is why the temporal results have slightly larger growth rates $\omega_{i}$ than those displayed in Fig. 2; this is related to the need of computing numerically $\mathrm{d}^{2} U / \mathrm{d} y^{2}$ and $\mathrm{d} C_{d} / \mathrm{d} y$ in the Orr-Sommerfeld-like equation. In italics, the growth rates obtained from Gaster's transformation are reported; the parameters imposed in each simulation are indicated with bold characters. The solutions for $R e=10^{9}$ coincide with those found using the inviscid equations.

\begin{tabular}{lcccccccc}
\hline \hline Theory & $R e$ & $\alpha_{r}$ & $\omega_{r}$ & $-\alpha_{i}$ & $\omega_{i}$ & $c_{r}$ & $c_{g}$ & $\operatorname{err}(\%)$ \\
\hline Temporal & 500 & $\mathbf{0 . 5 0}$ & 0.4778 & 0.0248 & 0.0254 & 0.9556 & 1.0245 & 0.54 \\
& 3450 & $\mathbf{0 . 5 0}$ & 0.4601 & 0.0413 & 0.0404 & 0.9202 & 0.9794 & 0.06 \\
& $10^{5}$ & $\mathbf{0 . 5 0}$ & 0.4514 & 0.0436 & 0.0421 & 0.9028 & 0.9661 & 0.63 \\
& $10^{9}$ & $\mathbf{0 . 5 0}$ & 0.4508 & 0.0451 & 0.0425 & 0.9016 & 0.9427 & 2.90 \\
Spatial & 500 & 0.4993 & $\mathbf{0 . 4 7 7 8}$ & 0.0248 & 0.0250 & 0.9569 & 1.0100 & 1.41 \\
& 3450 & 0.4990 & $\mathbf{0 . 4 6 0 1}$ & 0.0427 & 0.0404 & 0.9220 & 0.9471 & 3.30 \\
& $10^{5}$ & 0.4996 & $\mathbf{0 . 4 5 1 4}$ & 0.0449 & 0.0416 & 0.9109 & 0.9371 & 3.46 \\
& $10^{9}$ & 0.4993 & $\mathbf{0 . 4 5 0 8}$ & 0.0450 & 0.0411 & 0.9028 & 0.9143 & 3.01 \\
\hline \hline
\end{tabular}

The amplitude of the sensitivity functions, $\left|G_{U}(y)\right|$ and $\left|G_{C_{d}}(y)\right|$, in the spatial and temporal stability frameworks is of same order of magnitude (not shown here) since they are related through the complex group velocity $C_{g}$. It is found that $\left|G_{U}^{\text {temporal }}\right| \approx\left|C_{g}\right|\left|G_{U}^{\text {spatial }}\right|$ with $\left|C_{g}\right| \approx c_{g} \approx 1$ in the present case.

Obtaining and comparing results in the temporal and spatial stability frameworks, such as in Table I, is a good means to validate the sensitivity functions and to verify the accuracy of the computations of the adjoint stability equations.

${ }^{1}$ J. D. Ackerman and A. Okubo, "Reduced mixing in a marine macrophyte canopy," Funct. Ecol. 7, 305-309 (1993).

${ }^{2}$ M. Raupach, J. J. Finnigan, and Y. Brunei, "Coherent eddies and turbulence in vegetation canopies: The mixing-layer analogy,” Boundary Layer Meteorol. 78, 351-382 (1996).

${ }^{3}$ C. Py, E. De Langre, and B. Moulia, "The mixing layer instability of wind over a flexible crop canopy," C. R. Mec. 332(8), 613-618 (2004).

${ }^{4}$ R. Singh, M. M. Bandi, A. Mahadevan, and S. Mandre, "Linear stability analysis for monami in a submerged seagrass bed," J. Fluid Mech. 786(R1), 1-12 (2015).

${ }^{5}$ G. A. Zampogna, F. Pluvinage, A. Kourta, and A. Bottaro, “Instability of canopy flow,” Water Resour. Res. 52(7), 5421-5432, doi:10.1002/2016WR018915 (2016).

${ }^{6}$ S. Ikeda and M. Kanazawa, "Three-dimensional organized vortices above flexible water plants," J. Hydraul. Eng. 122(11), 634-640 (1996)

${ }^{7}$ M. C. Gambi, A. R. M. Nowell, and P. A. Jumars, "Flume observations on flow dynamics in Zostera marina (eelgrass) beds," Mar. Ecol.: Prog. Ser. 61, 159-169 (1990).

${ }^{8}$ J. E. Eckman, "The role of hydrodynamics in recruitment, growth and survival of Argopecten irradians and Anomia simplex within eelgrass meadows,” J. Exp. Mar. Biol. Ecol. 106, 165-191 (1987).

${ }^{9}$ R. E. Grizzle, F. T. Short, C. R. Newell, H. Hoven, and L. Kindblom, "Hydrodynamically induced synchronous waving of seagrasses: 'Monami' and its possible effects on larval mussel settlement," J. Exp. Mar. Biol. Ecol. 206, 165-177 (1996).

${ }^{10}$ J. J. Finnigan, "Turbulence in plant canopies," Annu. Rev. Fluid Mech. 32, 519-571 (2000).

${ }^{11}$ T. Asaeda, T. Fujino, and J. Manatunge, "Morphological adaptations of emergent plants to water flow," Freshwater Biol. 50, 1991-2001 (2005).

12 J. G. Duan, B. Barkdoll, and R. French, "Lodging velocity for an emergent aquatic plant in open channels," J. Hydraul. Eng. 132(10), 1015-1020 (2006).

${ }^{13}$ S. Patil and V. P. Singh, "Characteristics of monami wave in submerged vegetated flow," J. Hydrol. Eng. 15(3), 171-181 (2010).

${ }^{14}$ M. Ghisalberti and H. M. Nepf, "Mixing layers and coherent structures in vegetated aquatic flows,” J. Geophys. Res. 107(C2), 3-1-3-11, doi:10.1029/2001jc000871 (2002).

${ }^{15}$ M. Ghisalberti and H. M. Nepf, "The limited growth of vegetated shear layers," Water Resour. Res. 40(7), W07502, doi:10.1029/2003WR002776 (2004).

${ }^{16}$ M. Ghisalberti and H. M. Nepf, "Mass transport in vegetated shear flows," Environ. Fluid Mech. 5(6), 527-551 (2005).

${ }^{17}$ A. Bottaro, P. Corbett, and P. Luchini, "The effect of base flow variation on flow stability," J. Fluid Mech. 476, 293-302 (2003). 
${ }^{18}$ W. Brevis, M. García-Villalba, and Y. Niño, "Experimental and large eddy simulation study of the flow developed by a sequence of lateral obstacles," Environ. Fluid Mech. 14, 873-893 (2014).

${ }^{19}$ C. Canuto, M. Y. Hussaini, A. Quarteroni, and T. A. Zang, Spectral Methods in Fluid Dynamics (Springer-Verlag, Berlin, 1987).

${ }^{20}$ M. Le Bars and M. Worster, "Interfacial conditions between a pure fluid and a porous medium: Implications for binary alloy solidification," J. Fluid Mech. 550, 149-173 (2006).

${ }^{21}$ G. A. Zampogna and A. Bottaro, "Fluid flow over and through a regular bundle of rigid fibres," J. Fluid Mech. 792, 5-35 (2016).

${ }^{22}$ G. A. Zampogna, private communication (2016). The values of $\mathcal{K}_{11}$ and $\mathcal{K}_{22}$ arise from the solution of a local, microscopic problem which accounts for inertia through the porous medium. ${ }^{21}$ It is not unexpected that $\mathcal{K}_{11}$ approaches $\mathcal{K}_{22}$ as we leave the Stokes regime.

${ }^{23}$ G. S. Beavers and D. D. Joseph, "Boundary conditions at a naturally permeable wall," J. Fluid Mech. 30, 197-207 (1967).

${ }^{24}$ C. C. Mei and B. Vernescu, Homogenization Methods for Multiscale Mechanics (World Scientific Publishing Co. Pte. Ltd., Singapore, 2010).

${ }^{25}$ G. A. Zampogna and A. Bottaro, "The PELskin project-Part III: A homogenized model of flows over and through dense poroelastic media," Meccanica (published online). 